\title{
FINITE VARIETIES AND GROUPS WITH SYLOW $p$-SUBGROUPS OF LOW CLASS
}

\author{
ROLF BRANDL
}

(Received 24 October 1980)

Communicated by D. E. Taylor

\begin{abstract}
A finite variety is a class of finite groups closed under taking subgroups, factor groups and finite direct products. To each such class there exists a sequence $w_{1}, w_{2}, \ldots$ of words such that the finite group $G$ belongs to the class if and only if $w_{k}(G)=1$ for almost all $k$. As an illustration of the theory we shall present sequences of words for the finite variety of groups whose Sylow $p$-subgroups have class $c$ for $c=1$ and $c=2$.
\end{abstract}

1980 Mathematics subject classification (Amer. Math. Soc.): 20 D 10, 20 D 20, 20 E 10, 20 F 12.

\section{Introduction}

By a well known theorem of Zorn a finite group belongs to the class of finite nilpotent groups if and only if it satisfies almost all Engel conditions. This result can be viewed as an example of a description of a finite variety of groups by a sequence of laws. A class $f$ of finite groups is called a finite variety if it is closed under taking subgroups, factor groups and finite direct products. The central result on these classes reads as follows: given a finite variety $f$ then there exists a sequence of laws such that the finite group $G$ belongs to $f$ if and only if almost all of these laws hold identically in $G$. As an illustration of the theory we shall present sequences of laws in two variables for the classes of finite groups having abelian Sylow $p$-subgroups and Sylow $p$-subgroups of nilpotency class two. All groups considered in this paper are finite. 


\section{Finite varieties and their recognizability}

In this section the concept of a finite variety will be discussed.

DEFINITION. Let $f$ be a class of finite groups.

(a) $\mathfrak{f}$ is called a finite variety if $\mathfrak{t}$ is closed under taking subgroups, factor groups and finite direct products. Using P. Hall's well known closure operators we see that this just means $=\{Q, S, D\}$.

(b) $t$ is called $n$-recognizable if the following is true: "The finite group $G$ belongs to $f$ if and only if all of its $n$-generator subgroups belong to $f$.'

One easily verifies that finite varieties are just those formations which are closed under taking subgroups, so there are plenty of them. For example, the classes of finite abelian, nilpotent or supersoluble groups are finite varieties which are 2-recognizable (see Huppert (1967), p. 261 and p. 722). The reason why we have called our classes finite varieties lies in some similarity with varieties of groups. This is best illustrated by the following central result:

THEOREM A. Let be a finite variety of groups (which is n-recognizable). Then there exists a sequence $w_{1}, w_{2}, \ldots$ of words (in $n$ variables) such that the finite group $G$ lies in $f$ if and only if $w_{k}=1$ is a law in $G$ for almost all positive integers $k$.

The proof of Theorem A depends on the Oates-Powell Theorem (see Neumann (1967), p. 151) and on a result of G. Higman on finite groups in a variety generated by finitely many finite groups (see Neumann (1967), p. 18). It will appear in Brandl (to appear).

A typical illustration of Theorem $\mathrm{A}$ is a result due to Zorn which gives a description of the 2-recognizable finite variety of finite nilpotent groups by a sequence of laws in two variables. This sequence is given by the Engel words $w_{k}(x, y)=[x, k y]$. This paper is concerned with a special type of finite variety which we shall describe now.

Definition. Let $p$ be a prime and let $\sqrt{5}$ be a finite variety of $p$-groups. Then by Syl(ङ) we denote the class of all finite groups whose Sylow $p$-subgroups belong to 5 .

We have immediately:

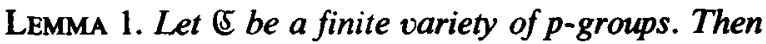

(a) $\operatorname{Syl}(\mathbb{E})$ is a finite variety,

(b) If (5) is n-recognizable then Syl(ङ) is n-recognizable. 
In the remainder of this note we shall discuss two examples of finite varieties.

\section{Groups with abelian Sylow p-subgroups}

In this chapter $p$ denotes some fixed prime number. Let $\mathbb{S}_{p}$ be the class of all finite groups having abelian Sylow $p$-subgroups. So $\mathbb{S}_{p}$ is a finite variety which is 2-recognizable. The object of this chapter is to describe this class by a sequence of laws. In order to do this we first introduce some notation.

Definition. (a) Let $\left\{p_{1}, p_{2}, \ldots\right\}$ be the set of all prime numbers different from $p$. For each positive integer $k$ we define $m_{k}:=\left(p_{1} \cdot \ldots \cdot p_{k}\right)^{k}$.

(b) Let $x, y$ be variables. For any positive integer $k$ we define the word $a_{k}(x, y):=\left[x^{m_{k}}, y^{m_{k}}\right]^{m_{k}}$.

The following result gives some information about groups satisfying some law $a_{k}(x, y)=1$.

LEMMA 2. Let $G$ be a finite group satisfying $a_{k}(G)=1$ for some positive integer $k$. Then $G$ has abelian Sylow p-subgroups.

Proof. Let $P \in \operatorname{Syl}_{p}(G)$ and let $x, y \in P$ be given. As $m_{k}$ and $|P|$ are coprime, we can find $x_{0}, y_{0} \in P$ with $x=x_{0}^{m_{k}}$ and $y=y_{0}^{m_{k}}$. Then, as $a_{k}(G)=1$, we have $1=a_{k}\left(x_{0}, y_{0}\right)=\left[x_{0}^{m_{k}}, y_{0}^{m_{k}}\right]^{m_{k}}=[x, y]^{m_{k}}$. Now, as $[x, y] \in P$, we can conclude that $[x, y]=1$ so $P$ is abelian.

In view of Theorem $A$ the question arises whether each group in $\mathfrak{S}_{p}$ satisfies almost all laws $a_{k}(x, y)=1$. This, however, is not the case in general, as the following shows.

EXAMPLE. Let $G=\operatorname{SL}\left(2, p^{2}\right)$ for some odd prime $p$. Let $c \in \operatorname{GF}\left(p^{2}\right)$ with $c^{2}=-4$. Furthermore, let $x=\left(\begin{array}{ll}1 & 1 \\ 0 & 1\end{array}\right)$ and $y=\left(\begin{array}{ll}1 & 0 \\ c & 1\end{array}\right)$. Then $[x, y]=\left(\begin{array}{cc}c-3 & c \\ 4 & 1\end{array}-c\right)$ has trace -2 and so has order $2 p$. In particular, we have $a_{k}(G) \neq 1$ for all $k$. But the Sylow $p$-subgroups of $G$ are abelian.

However, the prime $p=2$ plays a special role. We have

THEOREM B. Let $G$ be a finite group. Then the following are equivalent:

i) $G \in \widetilde{S}_{2}$,

ii) $G$ satisfies almost all laws $a_{k}(x, y)=1$. 
ProOF. In view of Lemma 2 we only need to show that groups with abelian Sylow 2-subgroups satisfy almost all $a_{k}(x, y)=1$. Let $G \in \widetilde{S}_{2}$. Then, by Walter (1969), there exist normal subgroups $K$ and $N$ of $G$ with $K \leqslant N$ such that $K$ and $G / N$ have odd order and $N / K \cong A \times E_{1} \times \cdots \times E_{s}$ with some abelian 2-group $A$ and simple groups $E_{i}$ possessing elementary abelian Sylow 2subgroups.

Let $k$ be such that the greatest odd integer which divides the order of $G$ also divides $m_{k}$. In proving $a_{k}(G)=1$ it suffices to show that for any elements $x, y$ of $G$ whose orders are powers of 2 the commutator $[x, y]$ has odd order. As $|G / N|$ is odd, we have $x, y \in N$. Without loss of generality we may assume that $K=1$. So $N \cong A \times E_{1} \times \cdots \times E_{s}$.

Let $x=x_{0} x_{1} \cdots x_{s}$ and $y=y_{0} y_{1} \cdots y_{s}$ with $x_{0}, y_{0} \in A$ and $x_{i}, y_{i} \in E_{i}$ for $1<i \leqslant s$. Then $[x, y]=\left[x_{1}, y_{1}\right] \cdots\left[x_{s}, y_{s}\right]$ as $A$ is abelian. For some $1 \leqslant i \leqslant s$ let $D=\left\langle x_{i}, y_{i}\right\rangle$. So $D$ is a dihedral group as $x_{i}$ and $y_{i}$ are involutions. As the Sylow 2-subgroups of $D$ are elementary abelian, the order of $\left[x_{i}, y_{i}\right]$ is odd. The result follows.

A result similar to Theorem B for $p$ odd is not known to the author. We just know by Theorem $A$ that some sequence of words in two variables does exist. However, if we restrict ourselves to $p$-soluble groups (we could do this by adding the sequence for the finite variety of $p$-soluble groups), we have a result for all $p$.

THEOREM B*. Let $G$ be p-soluble. Then the following conditions are equivalent:

i) G has abelian Sylow p-subgroups,

ii) $G$ satisfies almost all laws $a_{k}(x, y)=1$.

Proof. It suffices to show that each group $G \in \mathfrak{S}_{p}$ which is $p$-soluble satisfies almost all $a_{k}(x, y)=1$. By Huppert (1967), p. 691, $G$ has $p$-length one. Let $K$ and $N$ be normal subgroups of $G$ with $K \leqslant N$ such that $G / N$ and $K$ are $p^{\prime}$-groups and $N / K$ is an abelian $p$-group.

We can choose $k$ such that $\exp (G / N)$ and $\exp (K)$ both divide $m_{k}$. Then for any $x, y \in G$ we have $x^{m_{k}}, y^{m_{k}} \in N$. It follows that $\left[x^{m_{k}}, y^{m_{k}}\right] \in K$ and finally $a_{k}(x, y)=1$. This proves the result.

\section{4. $p$-soluble groups with Sylow $p$-subgroups of class two}

In this chapter $p$ denotes a fixed prime different from 3. We now introduce the laws we shall deal with. 
DEFINITION. For variables $x, y$ and some positive integer $k$ the word $b_{k}$ is defined by

$$
b_{k}(x, y):=\left[\left[x^{m_{k}}, y^{m_{k}}\right]^{m_{k}}, y^{m_{k}}\right]^{m_{k}}
$$

The following is a counterpart to Lemma 2:

LemMa 3. Let $G$ be a finite group satisfying $b_{k}(G)=1$ for some $k$. Then the Sylow p-subgroups of $G$ have class two.

Proof. Let $P \in \operatorname{Syl}_{p}(G)$ and let $x, y \in P$ be given. As in the proof of Lemma 2 choose $x_{0}, y_{0} \in P$ with $x=x_{0}^{m_{k}}$ and $y=y_{0}^{m_{k}}$. Then $1=b_{k}\left(x_{0}, y_{0}\right)=$ $\left[[x, y]^{m_{k}}, y\right]^{m_{k}}$. As $x, y \in P$ we have $\left[[x, y]^{m_{k}}, y\right]=1$, so $[x, y]^{m_{k}} \in C(y)$.

Now $[x, y] \in P$ and so the order of $[x, y]$ and $m_{k}$ are coprime. This yields $[x, y] \in C(y)$ and so $[x, y, y]=1$. So $P$ satisfies the second Engel condition and the result follows as $p \neq 3$.

In the proof of the main result of this section we shall utilize a corollary of Theorem B of Hall-Higman (see Gorenstein (1968), p. 359) which presumably is well known.

LEMMA 4. Let $G$ be a finite p-soluble group and let the Sylow p-subgroups of $G$ be of class two. Then

(a) $1_{p}(G)=1$ if $p \geqslant 5$,

(b) if $p=2$ and $O_{2^{\prime}}(G)=1$ then $O_{2,2^{\prime}, 2}(G) / O_{2,2^{\prime}}(G)$ is elementary abelian.

The purpose of this paragraph is to present a sequence of laws for the finite variety $\mathfrak{I}_{p}$ of all finite groups having Sylow $p$-subgroups of nilpotency class two. As $p \neq 3$ these classes are 2-recognizable by Neumann (1967), 34.31.

TheOREM C. Let $G$ be p-soluble, where $p$ is a prime different from 3. Then the following conditions are equivalent:

i) $G \in \mathfrak{I}_{p}$,

ii) $G$ satisfies almost all laws $b_{k}(x, y)=1$.

Proof. By Lemma 3, we only need to prove that a $p$-soluble group $G \in \mathfrak{I}_{p}$ satisfies almost all laws $b_{k}(x, y)=1$. If $p>5$ we know by Lemma 4 that $1_{p}(G)=1$ and the result follows immediately. So let $p=2$.

It suffices to show that for all 2-elements $x, y \in G$ the element $z=$ $\left[[x, y]^{m_{k}}, y\right]$ has odd order. We may assu e that $O_{2^{\prime}}(G)=1$ and need to show that $z=1$. Without loss of generality $G=\langle x, y\rangle$. We then have $G=O_{2,2^{\prime}, 2}(G)$. 
Let $R:=O_{2}(G)$ and define $W:=R / Z(R)$. Being generated by two involutions, $G / R$ is a dihedral group. We claim that $G / R$ centralizes $W$. Indeed, let $P \in \operatorname{Syl}_{p}(G)$. Then $Z(P) \leqslant C_{G}(R)=Z(R)$ by Gorenstein (1968), p. 228. If any involution in $G / R$ were to act nontrivially on $W$ then $P / Z(R)$ would be nonabelian and so the class of $P$ would exceed two. So $x R$ and $y R$ both centralize $W$ and we are done.

So $R / Z(R) \leqslant Z(G / Z(R))$ and $G / Z(R)$ possesses a normal 2-complement and has abelian Sylow 2-subgroups. This implies $[x, y]^{m_{*}} \in Z(R)$ for almost all $k$.

We now choose a 2-complement $D$ in $O_{2,2^{\prime}}(G)$ such that $[x, y]=r d$ for some $r \in Z(R)$ and $\langle d\rangle=D$. Let $Z(R)=C_{Z(R)}(D) \times[Z(R), D]=: R_{1} \times R_{2}$ and let $r=r_{1} r_{2}$ with $r_{i} \in R_{i}(i=1,2)$. Then $r_{1}$ is a 2-element and the order of $r_{2} d$ is odd. Moreover $\left[r_{1}, r_{2} d\right]=1$. So we have

$$
[x, y]^{m_{k}}=r_{1}^{m_{k}} \in R_{1} \quad \text { for almost all } k \text {. }
$$

By definition of $R_{2}$ we know that $D$ stabilizes the chain $R>Z(R) \geqslant R_{2}$. Hence $[R, D] \leqslant R_{2}$ and so $G / R_{2}$ is 2-nilpotent. Therefore,

$$
\left[[x, y]^{m_{k}}, y\right] \in R_{2} \text { for almost all } k \text {. }
$$

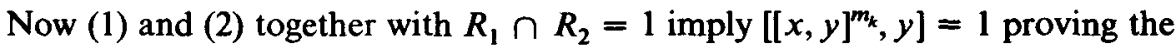
theorem.

\section{References}

R. Brandl (to appear), Zur Theorie der untergruppenabgeschlossenen Formationen: Endliche Varietäten, J. Algebra.

D. Gorenstein (1968), Finite groups (Harper \& Row, New York).

B. Huppert (1967), Endliche Gruppen I (Springer Verlag, Berlin).

H. Neumann (1967), Varieties of groups (Springer Verlag, Berlin).

J. Walter (1969), 'The characterization of finite groups with abelian Sylow 2-subgroups', Ann. of Math. (2) 89, 405-514.

Mathematisches Institut

Am Hubland

D-8700 Würzburg

Federal Republic of Germany 